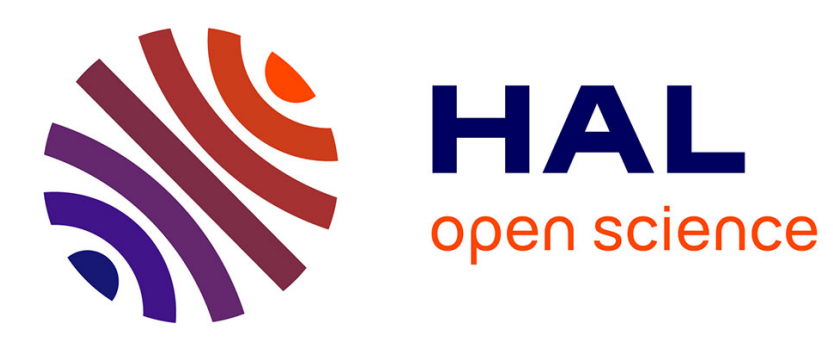

\title{
Specimen Preparation and Atom Probe Field Ion Microscopy of BSCCO-2212 Superconductors
}

\author{
D. Larson, P. Camus, J. Vargas, T. Kelly, M. Miller
}

\section{To cite this version:}

D. Larson, P. Camus, J. Vargas, T. Kelly, M. Miller. Specimen Preparation and Atom Probe Field Ion Microscopy of BSCCO-2212 Superconductors. Journal de Physique IV Proceedings, 1996, 06 (C5), pp.C5-271-C5-276. 10.1051/jp4:1996544 . jpa-00254423

\section{HAL Id: jpa-00254423 https://hal.science/jpa-00254423}

Submitted on 1 Jan 1996

HAL is a multi-disciplinary open access archive for the deposit and dissemination of scientific research documents, whether they are published or not. The documents may come from teaching and research institutions in France or abroad, or from public or private research centers.
L'archive ouverte pluridisciplinaire HAL, est destinée au dépôt et à la diffusion de documents scientifiques de niveau recherche, publiés ou non, émanant des établissements d'enseignement et de recherche français ou étrangers, des laboratoires publics ou privés. 


\title{
Specimen Preparation and Atom Probe Field Ion Microscopy of BSCCO-2212 Superconductors
}

\author{
D.J. Larson $*, * *(1)$, P.P. Camus**,***, J.L. Vargas**, T.F. Kelly*,**,*** and M.K. Miller**** \\ * Materials Science Program, University of Wisconsin, Madison, WI 53706, U.S.A. \\ ** Applied Superconductivity Center, University of Wisconsin, Madison, WI 53706, U.S.A. \\ *** Department of Materials Science and Engineering, University of Wisconsin, Madison, WI 53706, \\ U.S.A. \\ **** Metals and Ceramics Division, Oak Ridge National Laboratory, Oak Ridge, TN 37831-6376, U.S.A.
}

\begin{abstract}
Field ion specimens of $\mathrm{Bi}_{2} \mathrm{Sr}_{2} \mathrm{CaCu}_{2} \mathrm{O}_{x}$ (BSCCO) high temperature superconductor (HTS) materials have been prepared using a combination of three different preparation techniques: the method of sharp shards, electropolishing and ion milling. Field ion microscopy (FIM) has demonstrated that samples which exhibit the "striped"-image contrast characteristic of HTS materials can be successfully fabricated using this combination. FIM images have been obtained which show the striped-image contrast much clearer than any previously published images of $\mathrm{Pb}$-free $\mathrm{BSCCO}$. Preliminary atom probe (AP) chemical analysis of the material was also performed. Analytical electron microscopy was used to confirm the existence of both the correct crystallographic structure and nominal composition in the near-apex region of the specimen after preparation and FIM.
\end{abstract}

\section{INTRODUCTION AND BACKGROUND}

The use of high temperature superconductor (HTS) materials in various high current and/or high magnetic field applications requires a large value of the critical transport current density $\left(J_{C T}\right)$ [1]. $J_{C T}$ is the superconducting current density that can be transmitted over macroscopic distances in the material. At the present time, the grain boundary properties of polycrystalline HTS materials are believed to control the maximum $\mathrm{J}_{\mathrm{CT}}$ that can be sustained. This is due to the "weak links" (areas of reduced $\mathbf{J}_{\mathrm{CT}}$ ) that form at high-angle grain boundaries [2,3]. The problem of weak coupling at grain boundaries has been reduced in practice, however, by increasing the degree of texture in polycrystalline materials. This has been especially true for the BSCCO materials in the "2212" [4] and "2223" [5] phases and has lead to the fabrication of (001)-axis fiber-textured polycrystalline 2212 superconducting tapes with reasonably high $\mathrm{J}_{\mathrm{Cr}}$ values $\left(10^{5} \mathrm{~A} / \mathrm{cm}^{2}\right.$ at $4.2 \mathrm{~K}$ in fields up to $\left.20 \mathrm{~T}\right)[4,5]$. Due to the high degree to which the important properties of HTS materials depend on the grain boundary structure and chemistry, there is much interest in the development of advanced experimental techniques to study these structures.

Transmission electron microscopy (TEM) techniques have been the primary analytical tool for high resolution characterization of HTS materials and have shown that local compositional and structural defects can affect the superconducting properties [6-8]. However, the capabilities of current analytical instruments are reaching their limits and more powerful and complementary techniques are required for complete microstructural characterization. The distribution of oxygen at the grain boundaries is of particular interest [9]. The high spatial resolution and chemical analysis capabilities of atom probe field ion microscopy (APFIM) should make it an ideal technique to investigate BSCCO material. The following work is a preliminary study of the applicability of APFIM to BSCCO-2212 material.

(1) Current Address: Metals and Ceramics Division, Oak Ridge National Laboratory, Oak Ridge, TN 37831-6376, U.S.A. 


\section{APPROACHES TO SPECIMEN PREPARATION}

BSCCO single crystals were prepared by a directional solidification method [10] using $\mathrm{Bi}_{2} \mathrm{O}_{3}, \mathrm{SrCO}_{3}$, $\mathrm{CaCO}_{3}$ and $\mathrm{CuO}$ powders mixed in the 2212 cation stoichiometry. All materials used had a $99+\%$ cation purity. Plate-shaped crystals of 2212 were cleaved across their (001) planes to produce flakes up to several $\mathrm{mm}$ long and several tens of $\mu \mathrm{m}$ thick. These plates were then fragmented with a scalpel in an attempt to obtain rod-shaped shards. All BSCCO material used in this study was $\mathrm{Pb}$-free.

There are three approaches to APFIM specimen preparation: 1) chemical and electrochemical polishing, 2) ion beam milling and 3) the method of sharp shards. A properly formed APFIM sample should be in a cylindrically symmetric wire-type form with a radius of curvature at the apex of approximately $50 \mathrm{~nm}$ or less, have an approximately hemispherical "endcap" and have a shank angle as small as possible $\left(<45^{\circ}\right)$.

As in most APFIM applications, electropolishing was used initially for fabrication of specimens from the YBCO "123"-type superconductors [11]. Electropolishing has the advantage of being able to remove a relatively large amount of material faster and easier than other techniques. However, correct polishing conditions are sometimes difficult to obtain and may produce specimens with irregular surfaces resulting from preferential etching of the grain boundaries in the material [12]. Also, the formation of an insulating layer which impedes further electropolishing has been reported [12]. Although these problems may lead to inconsistency in fabrication of specimens, electropolishing has been used successfully by many groups to fabricate APFIM specimens from the 123 materials.

Ion beam milling has been used for preparation of various types of APFIM samples $[13,14]$ and is typically used for TEM sample preparation of HTS materials [15, 16]. A disadvantage of ion milling is the formation of a damaged/implanted layer of uncertain depth due to atom displacement from expected lattice sites and implantation of ions into the sample. However, a simulation of ion sputtering of a copper substrate using argon ions at 2 to $4 \mathrm{keV}$ results in a maximum implanted/damaged depth of only 3 to 5 $\mathrm{nm}$ [17]. APFIM has an added advantage over TEM in that field evaporation can be used to remove any amorphous or damaged layer prior to investigation. For some materials, ion milling may be the only feasible method of sample preparation. It also enables precise sculpting of the apex region of a sample [14], which can be used to modify the blade-shaped specimens often found when preparing HTS materials.

The method of sharp shards (MOSS) was developed in response to the idea that electropolishing and ion milling of HTS materials might introduce artifacts into these already complicated structures [18]. Using a high precision three-dimensional manipulator, suitably sharp fragments from a crushed wafer are mounted to a blunt metallic FIM specimen using electrically conductive epoxy. It was found that no subsequent specimen preparation was required for FIM imaging and a nearly pristine superconductor surface was produced [19]. The MOSS technique has the advantage of not modifying the chemical structure of a sample. It does, however, result in a fracture surface, the formation of which can structurally modify the underlying layers to an unknown depth and create microcracks. Also, much operator experience is required for proper selection of suitably sharp tips and the proper apparatus is also required. The feasibility of the MOSS method has been shown for YBCO and Pb-doped BSCCO materials. However, this study found its application irreproducible and too time consuming for routine use on $\mathrm{Pb}$-free BSCCO.

Initially, FIM investigation of " 123 " materials showed both randomly speckled images $[11,20]$ and images with striped contrast $[11,18,19,21]$. Striped-image contrast is predicted by simulations for layered perovskite structures $[18,22]$. Melmed et al. $[18,19]$ have suggested that the striped-image contrast is formed by the $\mathrm{Cu}-\mathrm{O}$ end planes imaging brightly in the " 123 " materials while Mesyats et al. have suggested that the yttrium atoms are brightly imaging [23]. Other possibilities have also been suggested [22].

Because of the difficulties encountered with the 123 materials and due to the multiphase characteristics of the initial BSCCO materials, few APFIM investigations of the newer materials have been performed. In addition, BSCCO materials caused additional difficulties, especially in specimen preparation. Thus, APFIM literature results on this material are limited and sometimes inconclusive.

BSCCO materials prepared by MOSS were found to break into less symmetrical and suitable fragments during specimen preparation. Initially, images were fragmentary in appearance $[19,24]$ and a number of years passed before high quality fully-striped images were obtained, and then only from $\mathrm{Pb}$ doped material [25]. In general, BSCCO specimens were found to be mechanically weaker than the "123" materials [19] and a much slower evaporation rate was proposed for any subsequent AP analysis [25]. It was found that $\mathrm{Pb}$-doped $\mathrm{BSCCO}$ seemed more resistant to fracture than undoped material and FIM images of twin boundaries have been observed in doped material [25]. 
Some measurements of doublet image rows in fragmentary 2212 images have been made which suggests that $\mathrm{Cu}-\mathrm{O}$ planes are producing the striped-image contrast [24]. It should be noted that all previous FIM investigations of BSCCO have used the MOSS method for sample preparation and electropolishing and ion miling of BSCCO for APFIM has not been reported.

\section{SPECIMEN PREPARATION AND APFIM OF BSCCO-2212}

Electropolishing of BSCCO-2212 was investigated and found to be feasible. The starting point for the electropolishing stage was a relatively blunt shard produced by the MOSS technique. It was found that BSCCO usually polished well with a (1 to 10$) \%$ solution of perchloric acid in butoxyethanol or glycerol solutions at 5 to $20 \mathrm{VDC}$ at room temperature. This is the same basic solution that has been used to electropolish the 123 materials [11] but its use on BSCCO materials has never been reported. In certain cases, the formation of an insulating layer which impedes electropolishing was observed.

Successful electropolishing of BSCCO enables even quite blunt shards (several $\mu \mathrm{m}$ initial radius) to be used as a starting point for APFIM sample preparation. A well-tapered BSCCO sample produced by electropolishing of a blunt shard is shown in Fig. 1. Smooth surfaces with nearly sharp enough apex radii (i.e. high voltage FIM images) were often, but not always, obtained along with controllable material removal. Micropolishing was also found to work as a stand alone method, as is illustrated by Fig. 2. This FIM image shows the striped-image contrast ( $a-b$ planes) much clearer than previously published images of $\mathrm{Pb}$-free BSCCO. All FIM images shown in this paper were obtained in (1 to 3 ) $\times 10^{-3} \mathrm{~Pa} \mathrm{~N}$.

Freshly-made BSCCO shards have a natural tendency to be blade-shaped due to their preferred cleavage plane. Electropolishing of such samples into a suitable APFIM geometry can be difficult because of this initial shape and due to the asymmetric electropolishing of the $\mathrm{a}-\mathrm{b}$ versus $\mathrm{c}$ direction. To alleviate this problem, ion milling can be employed to increase the cylindrical symmetry of blade-shaped specimens and to reduce the specimen apex radius.

Initial attempts were made to ion mill $\left(90^{\circ}\right.$ between the tip axis and beam) the sharpest shards that could be picked up using the MOSS method (no electropolishing step). Whether or not a very sharp sample could be made in this way depended primarily on the shape (shank angle) of the shard prior to milling. Specimens with radii on the order of 50-100 nm were achieved, but this was not straightforward and reproducible using ion milling alone because of the varying shapes of initial shards. It was apparent that ion milling might be significantly more effective if the shards had an approximately parallel shank (equiaxed cross section) before ion milling.

Ion milling of electropolished samples that were nearly sharp enough for APFIM (i.e. Fig. 1) was also investigated. Specimens have been successfully prepared using a combination of electropolishing and ion milling with radii ranging down to $\sim 15 \mathrm{~nm}$. The success rate when using both of the additional steps was markedly greater than using only the single MOSS step for the current work. An image of a sample fabricated by electropolishing and ion milling of a blunt shard is shown in Fig. 3. It should be noted that some samples never exhibit striped contrast at any voltage due to (1) failing before enough evaporation had occurred to expose non-damaged material or (2) being too blunt to allow the required field evaporation at reasonable voltages $(10-20 \mathrm{kV})$.

Specimens which did not exhibit striped-imaging contrast were analyzed by AP at Oak Ridge National Laboratory. A typical spectrum is shown in Fig. 4. The specimen temperature was $85 \mathrm{~K}$ and the data were collected in approximately $1 \times 10^{-5} \mathrm{~Pa} \mathrm{Ar}$. (The background vacuum for this instrument is $2 \times 10^{-9}$ $\mathrm{Pa}$.) The peaks in this spectrum show clearly the presence of $\mathrm{O}, \mathrm{Ca}, \mathrm{Cu}$ and $\mathrm{Sr}$, but no appreciable $\mathrm{Bi}$. In addition, the amount of $\mathrm{Cu}$ detected is much smaller than expected. A small amount of $\mathrm{Bi}$ is actually present in the spectrum as shown in Fig. 4 (mass range from 100 to 220). Bismuth is present in small amounts in both the single and double charge states, along with doubly-charged bismuth oxides. These results were duplicated from a second sample and suggest that the Bi is present in the sample but the AP analysis conditions are such that most of it is not being detected.

A possible scenario which explains the lack of $\mathrm{Bi}$ in the AP data is that field evaporation before and during initial FIM imaging is producing a corrugated surface structure with the striped-imaging species present in slightly protruding regions. Subsequent field evaporation of this type of structure using normal pulse fractions (15-20\%) will evaporate the protruding species first. This will eventually reveal the underlying elements, but the applied standing voltage may then lead to preferential evaporation of these elements during the time between voltage pulses. An abnormally large pulse fraction with a small DC voltage may be required in order to quantitatively analyze these materials. Clearly, further investigation is required. 


\section{CONFIRMATION OF 2212 PHASE}

In spite of the fact that striped-contrast images are being produced, the lack of certainty as to what type of specimen surface this image represents necessitates confirmation of the sample preparation method using a different technique. This was done using TEM in conjunction with energy dispersive $\mathrm{x}$-ray spectrometry (EDS) and diffraction analysis.

A specimen that was fabricated by electropolishing and ion milling and showed partially-striped image contrast in FIM was investigated in a Jeol 200CX TEM at $200 \mathrm{kV}$. The apex region of the sample is shown in Fig. 5 and is positioned with the c-axis parallel to the beam direction. The specimen was found to be quite blade-shaped; the measured tip radius in Fig. 5 is approximately $200 \mathrm{~nm}$ whereas the radius perpendicular to this direction was found to be $\sim 30 \mathrm{~nm}$. Beam damage due to TEM imaging is also apparent in Fig. 5.

Qualitative EDS analysis was used to determine if $\mathrm{Bi}$ was present in the field ion specimen in order to eliminate the possibility that $\mathrm{Bi}$ is being lost during specimen preparation or within the vacuum. Spectra were obtained at positions both near and away from the apex. The results from the two regions were essentially the same and the EDS spectra from the apex region ( $200 \mathrm{~nm}$ diameter beam) is shown in Fig. 6. An inordinately high copper signal is obtained due to the presence of the standard copper APFIM stub. The figure is scaled to the height of the Bi-M peak. There is unequivocal evidence for the presence of $\mathrm{Bi}$ in the material. Comparing the relative heights of the $\mathrm{Sr}-\mathrm{L}, \mathrm{Ca}-\mathrm{K}, \mathrm{Bi}-\mathrm{L}$ and $\mathrm{Sr}-\mathrm{K}$ peaks to the $\mathrm{Bi}-\mathrm{M}$ peak shows qualitative agreement with previous EDS results obtained for bulk 2212-phase material [6].

In order to identify the crystal structure of the material in the apex region of the specimen, selected area electron diffraction was used. The BSCCO-2212 material is easily identifiable in diffraction experiments due to the incommensurate modulation present in the crystallographic structure. The modulation occurs along the $\boldsymbol{b}$ axis and has a period equal to $4.7 \mathrm{~b}_{0}$ for the optimally-doped material having $T_{c}$ around $90 \mathrm{~K}[26]$. The selected area aperture was centered so that a region covering approximately $200 \mathrm{~nm}$ along the axis of the sample was selected. The [001] diffraction pattern (incident electron beam parallel to the $c$ axis) is shown in Fig. 7. The satellite spots parallel to $b^{*}$, which originate from the modulation, are easily visible in the pattern.

The pattern can be indexed by assigning to each diffraction spot a reciprocal lattice vector $K=$ $h a^{*}+k b^{*}+l c^{*}+m s^{*}$ where $a^{*}, b^{*}$ and $c^{*}$ are the reciprocal vectors of the real space lattice vectors $a, b$ and $c$, and $s^{*}$ is given by $(1 / \mathrm{s}) b^{*}$. The modulation period $\left(\mathrm{s} \mathrm{b}_{0}\right)$ was calculated to be $4.68 \mathrm{~b}_{0}$ from several measurements of the lengths of $b^{*}$ and $s^{*}$ in this pattern. Thus, the pattern indicates that the material in the apex region of this specimen is single-crystalline BSCCO-2212.

\section{CONCLUSIONS}

An APFIM sample preparation method for BSCCO-2212 has been developed using a combination of MOSS, electropolishing and ion milling techniques. This method has produced the highest quality $\mathrm{Pb}$-free BSCCO FIM images to date. A standard APFIM electropolishing apparatus and a standard ion mill are required. FIM results show striped-imaging contrast samples prepared using both electropolishing and electropolishing/ion milling. Field evaporation is often necessary, especially after ion milling has been done, in order to expose material that shows striped-image contrast. AP analyses of BSCCO-2212 specimens did not result in the correct stoichiometry and preferential evaporation of one or more elements seems likely.

Qualitative EDS results from a sample that showed partially-striped contrast suggest that, even after electropolishing and ion milling, the 2212 phase is present. Diffraction results show a good match in the incommensurate $b$-axis modulation known to occur in the 2212 phase material and this strongly supports the presence of this phase in the apex region of samples which show at least partially-striped contrast.

\section{Acknowledgments}

The authors acknowledge Drs. J.-L. Wang and R. J. Redwing for provision of BSCCO-2212 material. Helpful discussions with Drs. D. C. Larbalestier, J. B. Pawley, E. E. Hellstrom and S. E. Babcock are gratefully acknowledged. 
This research was supported by the National Science Foundation, Division of Materials Research under grant \#DMR-8911332 and by the Division of Materials Sciences, U. S. Department of Energy, under contract DE-AC05-96OR22464 with Lockheed Martin Energy Research Corp. Some of this research was conducted utilizing the Shared Research Equipment (SHaRE) User Program facilities at Oak Ridge National Laboratory.

\section{References}

[1] D. C. Larbalestier, Phys. Today 44, no. 6 (1991) 74.

[2] G. Deutscher and K. A. Müller, Phys. Rev. Lett. 59 (1988) 1745.

[3] D. Dimos, J. Mannhart and P. Chaudhari, Phys. Rev. B 41 (1990) 4038.

[4] K. Heine, N. Tenbrink and M. Thoner, Appl. Phys. Lett. 55 (1989) 2441.

[5] K. Sato, T. Hikata, H. Mukai, M. Ueyama, N. Shibuta, T. Kato, T. Masuda, M. Nagata, K. Iwata and T. Mitsui, IEEE Trans. Mag. 27 (1991) 1231.

[6] O. Eibl, Microscopy Research and Tech. 30 (1995) 218.

[7] S. E. Babcock and D. C. Larbalestier, Appl. Phys. Lett. 55 (1989) 393.

[8] J.-L. Wang, I-F. Tsu, X. Y. Cai, R. J. Kelly, M. D. Vaudin, S. E. Babcock and D. C. Laralestier, J Mater. Res. 11 (1996) 1.

[9] S. E. Babcock, X. Y. Cai, D. C. Larbalestier, D. H. Shin, N. Zhang, H. Zhang, D. L. Kaiser and Y Gao, Physica C 227 (1994) 183.

[10] D. B. Mitzi, L. W. Lombardo, A. Kapitulnik, S. S. Laderman and R. D. Jacowitz, Phys. Rev. B 41 (1990) 6564.

[11] G. L. Kellogg and S. S. Brenner, Appl. Phys. Lett. 51 (1987) 1851.

[12] G. Zaharchuk, L. von Alvensleben, M. Oehring and P. Haasen, J. de Phys. 49-C6 (1988) 471.

[13] M. Hellsing, Mat. Sci. Tech. 4 (1988) 824.

[14] K. B. Alexander, P. Angelini and M. K. Miller, J. de Phys. 50-C8 (1989) 549.

[15] R. Ramesh, G. Thomas, S. M. Green, Y. Mei, C. Jiang and H. L. Luo, Appl. Phys. Lett. 53 (1989) 1759.

[16] C. H. Chen, D. J. Werder, G. P. Espinosa and A. S. Cooper, Phys. Rev. B 39 (1989) 4686.

[17] D. J. Larson, Ph.D. Thesis, University of Wisconsin (1996).

[18] A. J. Melmed, R. D. Shull, C. K. Chiang and H. A. Fowler, Science 239 (1988) 117.

[19] A. J. Melmed, R. D. Shull and C. K. Chiang, J. de Phys. 49-C6 (1988) 459.

[20] A. Cerezo, C. R. M. Grovener, R. M. Hoyle and G. D. W. Smith, Appl. Phys. Lett. 52 (1988) 1020.

[21] G. L. Kellogg and S. S. Brenner, J. de Phys. 49-C6 (1988) 465.

[22] M. K. Miller and K. L. More, J. de Phys. 49-C6 (1988) 483.

[23] G. A. Mesyats, N. N. Syutkin, A. A. Ivchenko and E. F. Talantsev, J. de Phys. 49-C6 (1988) 477.

[24] Q. J. Gao, Z. X. Zhang, S. Y. Xu, S. Q. Feng and S. Z. Wang, J. de Phys. 50-C8 (1989) 487.

[25] A. J. Melmed, P. P. Camus, J. Vargas and D. C. Larbalestier, Appl. Surf. Sci. 67 (1993) 413.

[26] S. Kambe, K. Okuyama, S. Ohshima and T. Shimada, Physica C 250 (1995) 50.

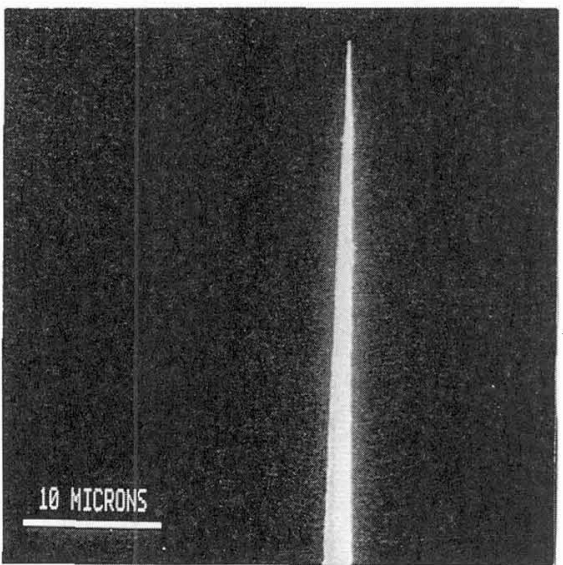

Figure 1. SEM image of a blunt shard after electropolishing. The apex radius is about $200 \mathrm{~nm}$.

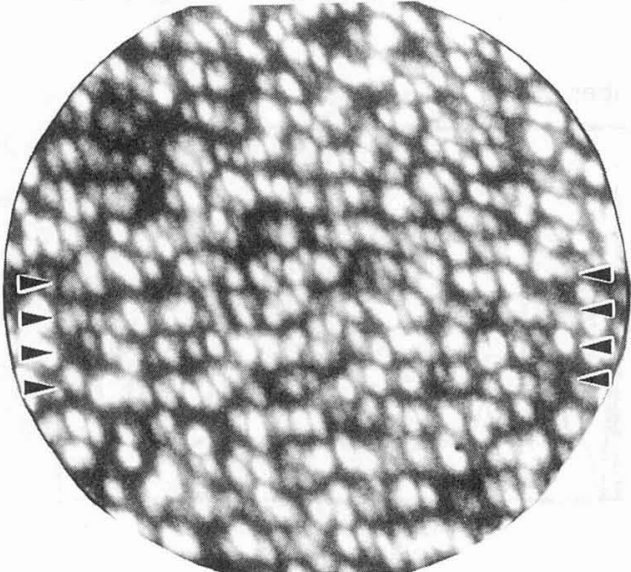

Figure 2. BSCCO FIM image of specimen fabricated by electropolishing only of a blunt shard. $\mathrm{V}=11 \mathrm{kV}$. 


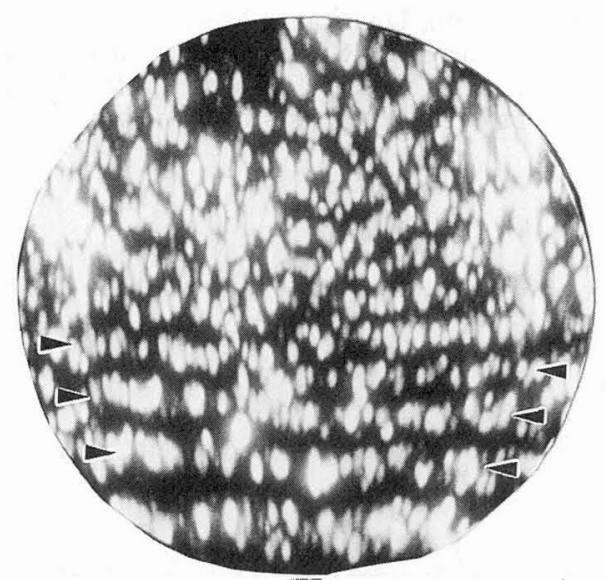

Figure 3. BSCCO FIM image of specimen fabricated by electropolishing and ion milling. $\mathrm{V}=9.9 \mathrm{kV}$.

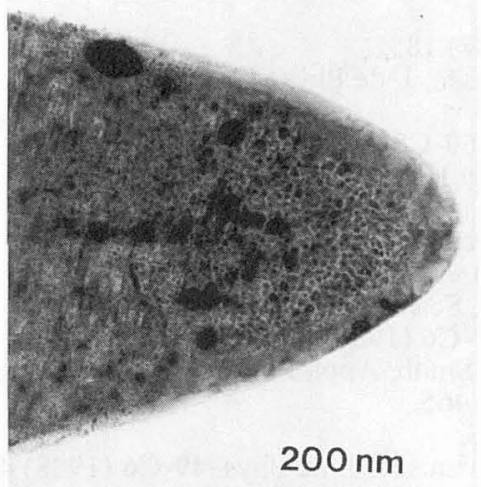

Figure 5. TEM image of apex region of BSCCO specimen.

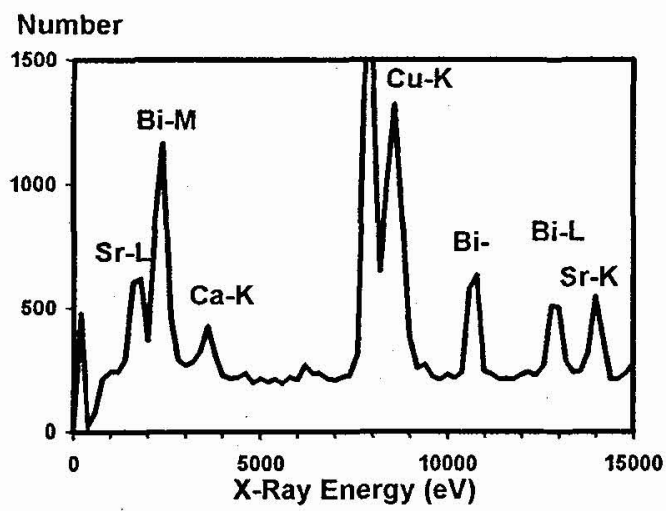

Figure 6. EDS spectrum from apex region of sample shown in Fig. 5.
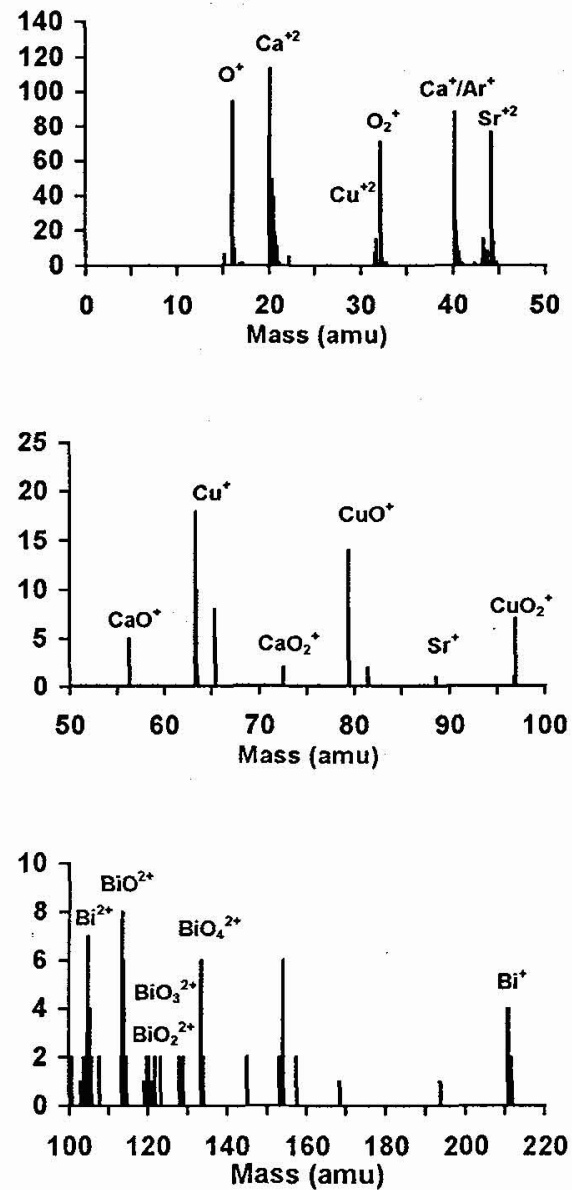

Figure 4. Atom probe spectrum of BSCCO-2212. $V=6-20$ $\mathrm{kV}$ with a $15-20 \%$ pulse fraction.

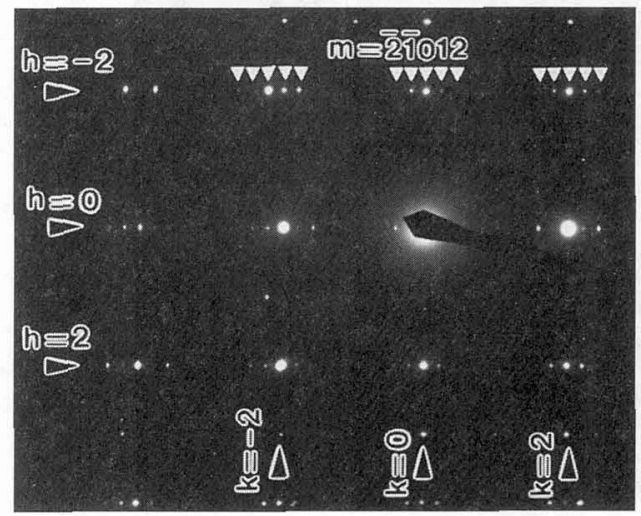

Figure 7. Diffraction pattern from apex region of sample shown in Fig. 5. 